\title{
PROGRAMAS DE PÓS-GRADUAÇÃO EM CONTABILIDADE: ANÁLISE DA PRODUÇÃO CIENTÍFICA E REDES DE COLABORAÇÃO
}

\author{
GRADUATE PROGRAMS IN ACCOUNTING: ANALYSIS OF PRODUCTION AND \\ SCIENTIFIC COLLABORATION NETWORKS
}

Harley Almeida Soares da Silva ${ }^{a}$; Diane Rossi Maximiano Reina ${ }^{b}$; Sandra Rolim Ensslin ${ }^{c}$; Donizete Reina ${ }^{d}$

\section{Resumo}

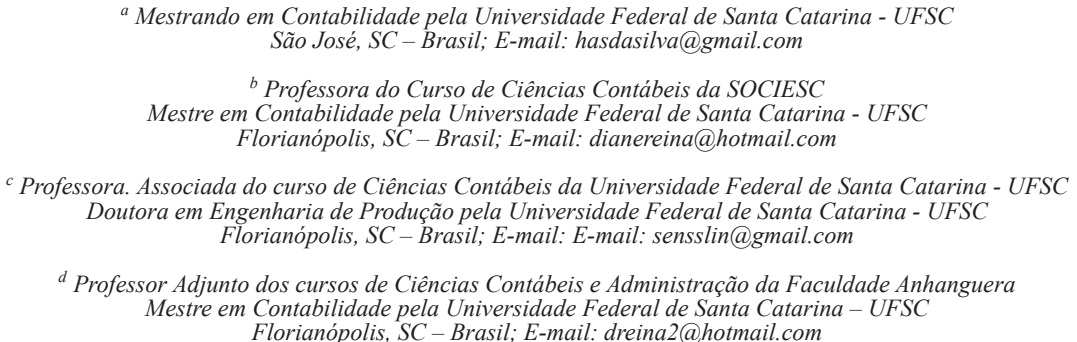

O presente estudo teve como objetivo mapear as redes de colaboração científica, em periódicos, dos docentes vinculados aos programas de Pós-Graduação em Contabilidade no triênio 20072009 , identificando a quantidade de atores, vínculos, interações e grau de centralização das redes de colaboração dos programas. Trata-se de uma pesquisa descritiva, com abordagem qualitativa e quantitativa, documental, com dados secundários. Constatou-se que: (i) os programas da USP e da FURB possuem as maiores médias de quantidade de atores; (ii) há uma tendência evolutiva dos programas FUCAPE, FURB, PUC SP, UFBA, UFPE, UFRJ, UFSC e UNISINOS no que diz respeito à quantidade de vínculos das redes de colaboração. Conclui-se que a maioria dos programas tem contribuído para a evolução das pesquisas e publicações científicas na área contábil, e, para tanto, tem-se utilizado parcerias colaborativas no desenvolvimento de suas pesquisas.

Palavras-chave: Redes de Colaboração; Redes Sociais; Programas de Pós-Graduação; Produção Científica de Docentes; Currículo Lattes.

\section{Abstract}

This study aimed at mapping the scientific collaboration networks, in journals, of professors connected with the Postgraduate Program in Accounting in the period 2007-2009, identifying the number of actors, relations, interactions and degree centralization of collaborative networks of programs. It is a descriptive research with quantitative and qualitative approach, documentary, using secondary data. It was noted that: (i) USP and FURB programs have the highest average number of actors, (ii) there is an evolutionary trend in the programs of $F U$ CAPE, FURB, PUC SP, UFBA, UFPE, UFRJ, UFSC and UNISINOS regarding the amount of bonds of collaboration networks. It was concluded that most programs have contributed to the evolution of research and scientific publications in accounting, and, therefore, have been used for collaborative partnerships in the development of their research.

Keywords: Collaboration Networks; Social Networks; Postgraduate Programs; Scientific Production of Professor; Curriculum Lattes. 


\section{INTRODUÇÃO}

O desenvolvimento de pesquisas científicas e sua divulgação são importantes meios de construção e disseminação de conhecimentos. Como fonte de construção de conhecimento, as instituições de ensino superior representam o campo perfeito para a construção de novos saberes. Nesse contexto, encontram-se inseridos os programas de pós-graduação, como instrumentos de formação de pesquisadores, mestres e doutores, que contribuirão para o desenvolvimento de conhecimentos (LEITE FILHO, 2006).

A evolução das Ciências Contábeis no Brasil foi impulsionada pela expansão dos programas de Pós-Graduação lato sensu e stricto sensu (BEUREN et al., 2009), resultando em um gradativo crescimento nas pesquisas e publicações científicas. No entanto, parte desse crescimento é decorrente do empenho do corpo docente e discente, e da pressão exercida pela Coordenação de Aperfeiçoamento de Pessoal de Nível Superior (CAPES), para que os programas desenvolvam publicações científicas relevantes, sendo esse um dos critérios para a avaliação dos programas pelo Ministério da Educação (SILVA, OLIVEIRA e RIBEIRO FILHO, 2005; LEITE FILHO, 2006).

A formação de redes de colaboração entre pesquisadores representa um meio para a formação de novos conhecimentos científicos. Segundo Cruz et al. (2010, p. 2), "a colaboração entre pesquisadores possibilita o compartilhamento de recursos informacionais, tecnológicos, além de experiências, ideias entre outras trocas".

Nessa perspectiva, com o intuito de investigar a produção científica e as redes de colaboração dos docentes dos programas de Pós-Graduação em Contabilidade, emerge a seguinte pergunta: Qual a quantidade de atores, vínculos, interações e grau de centralização das redes de colaboração dos docentes dos programas de Pós-Graduação em Contabilidade no contexto brasileiro? Para responder a tal questionamento, o presente artigo tem como objetivo mapear as redes de colaboração científica, em periódicos, dos docentes vinculados aos programas de Pós-Graduação em Contabilidade no triênio 2007-2009, identificando a quantidade de atores, vínculos, interações e grau de centralização das redes de colaboração dos programas.

Embora existam estudos que se propõem a investigar a produção científica e outros que investigam as redes de colaboração (LYRIO, BORBA e COSTA, 2007; MANAINARDES et al., 2008; BORBA e MURCIA, 2006; BEUREN, SCHLINDWEIN e PASQUAL, 2007; SOUZA et al., 2008; ENSSLIN e SILVA, 2008; BEUREN et al., 2009), esta pesquisa se justifica, uma vez que não se encontrou nenhum estudo com o propósito de investigar a produção científica e as redes de colaboração dos docentes vinculados aos programas de Pós-Graduação em Contabilidade no triênio 2007-2009.

A relevância da pesquisa pode ser confirmada por apresentar as redes de colaboração dos programas de Pós-Graduação em Contabilidade e avaliar o desempenho desses programas no que se refere à disseminação de conhecimento por meio das produções científicas publicadas em periódicos, evidenciando assim sua colaboração como instrumento de formação de pesquisadores que irão gerar e disseminar novos conhecimentos.

Este estudo delimita seu escopo na investigação dos artigos completos publicados em periódicos e nas redes de colaboração dos docentes vinculados aos programas de Pós-Graduação em Contabilidade, reconhecidos pela Coordenação de Aperfeiçoamento de Pessoal de Nível 
Superior (CAPES), referentes aos anos de 2007 a 2009, disponível no Currículo Lattes de cada docente.

O presente estudo está organizado em cinco seções: após esta primeira seção de caráter introdutório, apresenta-se, na segunda, o referencial teórico sobre Produção Científica e Redes de Colaboração; na terceira seção, apresenta-se a metodologia da pesquisa, subdividida em enquadramento metodológico, amostra e procedimentos para coleta e análise dos dados; na quarta, apresentam-se os resultados da pesquisa; na quinta e última seção, apresentam-se as considerações finais. Por fim, elencam-se as referências utilizadas na pesquisa.

\section{REFERENCIAL TEÓRICO}

\subsection{Produção científica}

Para que ocorra o desenvolvimento científico, a ciência depende de dois fatores primordiais: a pesquisa científica e a divulgação científica (SOARES, EBSEN, PFITSCHER, 2010).

Segundo Marion e Marion (1998, p. 3), "Pesquisa significa busca, indagação e investigação. Pesquisar é produzir conhecimento, formar conhecimento". Nesse sentido, a pesquisa e a divulgação científica são meios que possibilitam a construção e a disseminação de conhecimentos nas diversas áreas.

As instituições de ensino superior possuem um importante papel para o avanço da ciência: como fonte de construção de conhecimento o desafio das universidades é a produção de conhecimento próprio com qualidade formal e política capaz de promover o desenvolvimento. Isso só é possível mediante a realização de pesquisa como estratégia de geração de conhecimento e de promoção da cidadania (TEIXEIRA et al., 2007). Nesse contexto, podem-se destacar os programas de pós-graduação por serem instrumentos de formação de pesquisadores, mestres e doutores que contribuirão para a produção de novos conhecimentos (LEITE FILHO, 2006).

Nos últimos anos, as Ciências Contábeis vêm evoluindo no Brasil, impulsionada pelo surgimento de novos cursos de pós-graduação lato e stricto sensu (BEUREN et al., 2009). Como consequência, espera-se a ampliação no número de publicações científicas, pois o objetivo da maioria dos programas de mestrado e doutorado em Contabilidade é a formação de pesquisadores e docentes.

Para garantir a qualidade dos programas de pós-graduação, a avaliação da produtividade científica é um dos principais elementos, por possibilitar à CAPES avaliar o desempenho das instituições por meio de sua produtividade intelectual. No entanto, para que a CAPES possa efetuar a avaliação dos programas, é imprescindível que a produção intelectual seja publicada, e a publicação em periódicos tem sido a forma mais utilizada para divulgação das pesquisas científicas. Diante disso, a CAPES desenvolveu o Qualis que é um conjunto de procedimentos para estratificação da produção intelectual desenvolvida pelos programas.

A qualidade das publicações é auferida de acordo com a classificação dos periódicos, que passam por um processo de avaliação anual e são enquadrados por estratos indicativos de qualidades sendo: A1 o mais elevado, seguido de A2; B1; B2; B3; B4; B5; e, por último, C com pontuação zero. 
Quanto às pesquisas relacionadas que se propõem a investigar a produção científica, pode-se mencionar o estudo realizado por Theóphilo e Iudícibus (2005). Estes desenvolveram uma análise crítico-epistemológica da produção científica em Contabilidade no Brasil. Foram analisados 238 trabalhos, publicados entre 1994 e 2003, dentre eles teses e dissertações dos programas de pós-graduação recomendados pela CAPES, artigos de periódicos e trabalhos de congressos com melhores avaliações pelo sistema Qualis da CAPES.

Borba e Murcia (2006) pesquisaram as características das revistas de Contabilidade publicadas em língua inglesa constantes no Portal de Periódicos da CAPES, com o objetivo de identificar e segmentar as revistas acadêmicas de Contabilidade em língua inglesa do portal da CAPES.

Beuren, Schlindwein e Pasqual (2007) buscaram identificar o perfil das pesquisas em Controladoria publicadas nos anais do EnANPAD e do Congresso USP de Controladoria e Contabilidade no período de 2001 a 2006. Já Ensslin e Silva (2008), com o objetivo de identificar o perfil das produções científicas nos congressos de Contabilidade sediados pelos departamentos de Contabilidade da USP e da UFSC, desenvolveram uma pesquisa com o objetivo de sistematizar as publicações dos Congressos USP de Controladoria e Contabilidade e Iniciação Científica no ano de 2004 e comparar com a produção do Congresso UFSC de Controladoria e Finanças e Iniciação Científica no ano de 2007. Nessa mesma linha, Manainardes et al. (2008) analisaram a produção científica publicada nos anais do EnANPAD e do Encontro de Marketing (EMA) no período de 2003 a 2006, com o objetivo de analisar a produção científica na área de marketing de serviços.

Lyrio, Borba e Costa (2007) pesquisaram o perfil metodológico da produção científica relacionada à área de Controle Gerencial, publicada nos período de 2000 a 2004, nos periódicos classificados com conceito "A" no sistema Qualis/CAPES de 2004, nas áreas de Administração e Contabilidade.

\subsection{Redes de colaboração}

A formação de redes de colaboração entre pesquisadores representa um meio para a formação de novos conhecimentos científicos. Segundo Cruz et al. (2010, p. 2), "a colaboração entre pesquisadores possibilita o compartilhamento de recursos informacionais, tecnológicos, além de experiências, ideias entre outras trocas".

Bastos e Borges-Andrade (2004, p. 69) chamam a atenção para a importância dos atores sociais no processo de construção do conhecimento, diante da "existência de esquemas interpretativos ancorados em diferentes valores sociais".

Para Kuhn (1970), o crescimento da produção científica está altamente relacionado com o desenvolvimento da organização social científica. Nesse contexto, as redes de colaboração científica representam os vínculos entre pesquisadores e instituições no que diz respeito à produção científica. No Quadro 1, evidenciam-se os elementos básicos que compõem as redes de colaboração. 


\begin{tabular}{|l|}
\hline $\begin{array}{l}\text { Nós ou atores: pessoas, grupo de pessoas ou instituições que se agrupam com um objetivo comum, cada ator } \\
\text { representa um nó na rede. }\end{array}$ \\
\hline $\begin{array}{l}\text { Vínculos: são os laços firmados entre os atores, os vínculos são representados pelas linhas que ligam os nós na } \\
\text { representação gráfica da rede. }\end{array}$ \\
\hline Fluxo: direção do vínculo firmado entre os atores, representado por uma seta que indica o sentido da interação. \\
\hline $\begin{array}{l}\text { Nós soltos: atores que não estabelecem qualquer tipo de fluxo na rede, indicando a ausência de vínculos dentro } \\
\text { da rede. }\end{array}$ \\
\hline Grau de Centralidade: número de nós a que um determinado ator está diretamente ligado. \\
\hline $\begin{array}{l}\text { Grau de Centralização: condição em que o ator exerce papel claramente central na rede ao estar ligado aos } \\
\text { demais nós, aos quais precisam passar pelo nó central para se ligarem uns aos outros. }\end{array}$ \\
\hline Interações: quantidade de relações estabelecidas pelo ator e os nós. \\
\hline Grau de Saída: soma das interações que o ator tem com os outros nós da rede. \\
\hline Grau de Entrada: soma das interações que os outros nós da rede tem com o ator. \\
\hline
\end{tabular}

Quadro 1: Definições dos elementos básicos das redes de colaboração

Fonte: Adaptado de Alejandro e Norman, 2005

As redes de colaboração apresentam-se como alternativas para uma maior difusão do conhecimento científico, em que os laços entre os pesquisadores e as instituições são fortalecidos por meio da colaboração na produção científica (BEUREN et al., 2009; ESPEJO et al., 2009; WALTER et al., 2009). A compreensão das relações entre pesquisadores apresenta-se como um dos principais elementos para a compreensão do perfil da produção científica desenvolvida pelos docentes, pois permite identificar as oportunidades de pesquisa e parceria científica, além de fortalecer a comunicação entre as instituições de ensino e a pesquisa.

Quanto às pesquisas relacionadas que se propõem a investigar as redes de colaboração científica, pode-se mencionar a realizada por Beuren et al. (2009), com o objetivo de analisar as redes de pesquisa dos egressos do curso de Doutorado em Ciências Contábeis da FEA/USP. Já Souza et al.(2008) analisaram a produção científica das instituições de ensino superior da área de Ciências Contábeis e seus pesquisadores, pela análise de 657 artigos, sendo 268 artigos dos anais do Congresso USP de Controladoria e Contabilidade, 316 do EnANPAD, de 2006 e 2007, e 47 da ANPCONT, bem como 26 do EnEPQ, de 2007.

O estudo de Guimarães et al.(2009) buscou analisar a formação de redes de colaboração entre programas de Pós-Graduação em Administração, onde os autores identificaram que a rede pesquisada apresentou-se pouco densa, com relações esparsas e em sua maioria fracas entre programas, e que a localização geográfica do programa e as linhas de pesquisa adotadas são fatores importantes na definição da estrutura da rede. O estudo de Nascimento e Beuren (2011) também identificou a existência de relações esparsas, fracas, e com baixa densidade entre os programas de Pós-Graduação em Ciências Contábeis no triênio de 2007-2009 e concluiu que a USP exerce a centralidade na rede estabelecida entre os programas.

Diferentemente dos trabalhos de Guimarães et al.(2009) e de Nascimento e Beuren (2011) a presente pesquisa visa identificar elementos das redes de colaboração (como a quantidade de atores, vínculos, interação, e grau de centralização) com o intuito de demonstrar indicativos que apontem para a construção do conhecimento e evolução das Ciências Contábeis advindos da 
interação entre os pesquisadores proporcionada pela rede de colaboração. Ou seja, das parcerias estabelecidas nas publicações em periódicos científicos, dentro do contexto dos programas de Pós-Graduação em Ciências Contábeis, uma vez que acredita-se que esses programas exerçam importante contribuição para evolução da Contabilidade no contexto brasileiro, e que as parcerias nas publicações podem potencializar a troca de informações e conhecimento entre os pesquisadores.

\section{METODOLOGIA DA PESQUISA}

Nesta seção, são descritos: (i) o enquadramento metodológico da pesquisa; (ii) a amostra; e (iii) os procedimentos para coleta e análise dos dados.

\subsection{Enquadramento metodológico}

No que concerne à natureza do objetivo, este trabalho possui caráter descritivo (GIL, 2010), uma vez que visa mapear a produção científica e as redes de colaboração dos docentes vinculados aos programas de Pós-Graduação em Contabilidade, a partir da investigação da quantidade de atores que compõem as redes; da pontuação obtida no Qualis /CAPES; da quantidade de vínculos traçados em cada programa; da quantidade de interações de pesquisa estabelecidas nas redes; e do grau de centralização das redes.

No que tange à natureza do artigo, esta pesquisa se enquadra como um estudo prático e tem as características de um estudo Survey (TRIPODI; FELLIN; MEYER, 1981), tendo em vista que a partir dos resultados encontrados é possível conhecer o comportamento das redes de colaboração dos docentes vinculados aos programas de Pós-Graduação em Contabilidade, no período mapeado.

Considerando-se o problema de pesquisa estabelecido, esta pesquisa faz uso da abordagem qualitativa e quantitativa (RICHARDSON, 1999). A pesquisa é qualitativa por examinar e refletir sobre a produção científica dos docentes pelo viés da rede de colaboração para obter um entendimento do comportamento das redes de colaboração científica dos docentes vinculados aos programas de Pós-Graduação em Contabilidade no triênio 2007-2009; a abordagem quantitativa é verificada através da construção e da análise das redes de colaboração científica por meio do software UCINET ${ }_{\circledast}$ 6, do cálculo dos coeficientes de Correlação de Pearson entre as variáveis de pesquisa e das análises estatísticas descritivas por meio do software SPSS 19.

O procedimento técnico adotado foi o da pesquisa documental (FACHIN, 2005), a coleta de dados foi realizada por intermédio do Currículo Lattes dos docentes vinculados aos programas de Pós-Graduação em Contabilidade, configurando-se assim como dados secundários (RICHARDSON, 1999).

\subsection{População e amostra}

A população desta pesquisa é composta pelos docentes vinculados aos programas de 
Pós-Graduação stricto sensu em Contabilidade, Ciências Contábeis e Controladoria, conforme lista de cursos recomendados e reconhecidos pela CAPES (Quadro 2). A relação dos docentes foi obtida no Caderno de Indicadores, na ficha de corpo acadêmico, vínculo e formação no triênio 2007-2009. Foram objetos de análise todos os docentes apontados pelas fichas dos programas, ou seja, aqueles que possuem vínculo permanente, ou colaborador e ou visitante.

\begin{tabular}{|l|l|c|c|}
\hline \multicolumn{1}{|c|}{ Curso } & Nível & Conceito (2007/2008/2009) \\
\hline FUCAPE & \multicolumn{1}{|c|}{ Instituição } & $\mathrm{M} / \mathrm{D} / \mathrm{F}$ * & $4-4-5$ \\
\hline FURB & Ciências Contábeis & $\mathrm{M} / \mathrm{D}$ & 4 \\
\hline PUC/SP & Ciências Contábeis & $\mathrm{M}$ & 3 \\
\hline UERJ & Ciências Contábeis e Atuariais & $\mathrm{M}$ & 3 \\
\hline UFAM & Ciências Contábeis & $\mathrm{F}$ & 3 \\
\hline UFBA & Contabilidade e Controladoria & $\mathrm{M}$ & 3 \\
\hline UFC & Contabilidade & $\mathrm{M} / \mathrm{F}$ & 3 \\
\hline UFES & Administração e Controladoria & $\mathrm{M}$ & 3 \\
\hline UFMG & Ciências Contábeis & $\mathrm{M}$ & 4 \\
\hline UFPE & Ciências Contábeis & $\mathrm{M}$ & 3 \\
\hline UFPR & Ciências Contábeis & $\mathrm{M}$ & 3 \\
\hline UFRJ & Contabilidade & $\mathrm{M}$ & 4 \\
\hline UFSC & Ciências Contábeis & $\mathrm{M} / \mathrm{D}$ & 4 \\
\hline UNB-UFPB-UFRN & Contabilidade & $\mathrm{M}$ & 4 \\
\hline UniFECAP & Contabilidade & $\mathrm{M}$ & 4 \\
\hline UNISINOS & Ciências Contábeis & $\mathrm{F}$ & 4 \\
\hline UPM & Ciências Contábeis & $\mathrm{M} / \mathrm{D}$ & 4 \\
\hline USP & Ciências Contábeis & $\mathrm{M}$ & \\
\hline USP/RP & Controladoria e Contabilidade & $\mathrm{T}$ \\
\hline
\end{tabular}

*Legenda: M- Mestrado; D- Doutorado; F- Mestrado Profissionalizante.

Quadro 2: Relação de Cursos recomendados pela CAPES

Fonte: CAPES (2011a)

Entre os programas demonstrados no Quadro 2, o de mestrado em Ciências Contábeis da UFES e o de doutorado na FUCAPE não foram analisados, uma vez que estes, até a data do desenvolvimento desta pesquisa, não possuíam ainda avaliação de seus indicadores. Optou-se também em manter o programa de mestrado em Administração e Controladoria da UFC, tendo em vista que apresentava dados referentes ao ano de 2009.

\subsection{Procedimentos para coleta e análise dos dados}

Os dados referentes à produção científica dos docentes foram extraídos dos Currículos Lattes disponibilizados na Plataforma Lattes do Conselho Nacional de Desenvolvimento Científico e Tecnológico (CNPq), nos dias 25 e 26/01/2011. Os conceitos dos periódicos foram obtidos pelo estrado Qualis/CAPES da área de Administração, Contabilidade e Turismo, por meio do WebQualis. Os periódicos foram estratificados e conceituados de acordo com a seguinte pontuação: A1 - 100 pontos, A2 - 80 pontos, B1 - 60 pontos, B2 - 50 pontos, B3 - 30 pontos, B4 - 20 pontos, B5 - 10 pontos e C - 0 ponto (CAPES, 2011). São objetos de análise apenas as publicações em periódicos realizadas no período de 2007 a 2009, registrados no Lattes dos docentes.

As redes de colaboração apresentadas no presente artigo foram detectadas nas publicações em periódicos, correspondentes ao triênio 2007-2009, conforme apresentado nos 
Lattes dos docentes.

Para a construção e análise das redes de colaboração científica, foi utilizado o software UCINET® 6; já para o cálculo dos coeficientes de Correlação de Pearson entre as variáveis de pesquisa e as análises das estatísticas descritivas apresentadas no presente artigo, utilizou-se o software SPSS 19.

\section{APRESENTAÇÃO DOS RESULTADOS}

Ao mapear as redes de colaboração científica estabelecidas pelos docentes dos programas analisados, detectaram-se os valores correspondentes às seguintes variáveis em cada programa: (i) quantidade de atores que compõem as redes; (ii) pontuação obtida no Qualis /CAPES; (iii) quantidade de vínculos traçados em cada programa; (iv) quantidade de interações de pesquisa estabelecidas nas redes; e (v) grau de centralização das redes.

A variável pontuação no Qualis/CAPES (QUA), quantidade de vínculos (VINC) e quantidade de interações (INTE) foram detectadas por docente. Dessa forma, foi possível verificar a existência de uma forte correlação positiva entre elas, com significância ao nível de 0,01 (Tabela 1), ou seja, uma correlação bicaudal nos três períodos compreendidos pela análise.

Tabela 1: Correlação entre as variáveis detectadas por docente

\begin{tabular}{|c|c|c|c|c|c|c|c|c|c|c|}
\hline & & \multicolumn{3}{|c|}{2007} & \multicolumn{3}{|c|}{2008} & \multicolumn{3}{|c|}{2009} \\
\hline & & QUA & VINC & INTE & QUA & VINC & INTE & QUA & VINC & INTE \\
\hline \multirow{3}{*}{ QUA } & Correlação de Pearson & 1 & & & 1 & & & 1 & & \\
\hline & Significância (bicaudal) & & & & - & & & - & & \\
\hline & $\mathrm{N}$ & 188 & & & 216 & & & 259 & & \\
\hline \multirow{3}{*}{ VINC } & Correlação de Pearson &, $721 * *$ & 1 & &, $704 * *$ & 1 & &, $729 * *$ & 1 & \\
\hline & Significância (bicaudal) &, 000 & & &, 000 & - & &, 000 & - & \\
\hline & $\mathrm{N}$ & 188 & 188 & & 216 & 216 & & 259 & 259 & \\
\hline \multirow{3}{*}{ INTE } & Correlação de Pearson &, $746 * *$ &, $954 * *$ & 1 &, $744 * *$ &, $943 * *$ & 1 &, $756 * *$ &, $968 * *$ & 1 \\
\hline & Significância (bicaudal) &, 000 &, 000 & &, 000 &, 000 & - &, 000 &, 000 & - \\
\hline & $\mathrm{N}$ & 188 & 188 & 188 & 216 & 216 & 216 & 259 & 259 & 259 \\
\hline
\end{tabular}

Legenda: $\mathrm{N}$ - número de docentes no período.

Fonte: Dados da Pesquisa

Os resultados obtidos na Tabela 1 apontam para uma forte correlação positiva entre a pontuação obtida pelos docentes no Qualis /CAPES (QUA) com a quantidade de vínculos (VINC) que os mesmos estabelecem com outros autores ou co-autores nas publicações de artigos em periódicos. A pontuação no Qualis /CAPES (QUA) também apresentou uma correlação positiva significativa com a quantidade de interação (INTE), que consiste na quantidade de vezes que o docente estabelece uma parceria com outros autores ou co-autores.

Portanto acredita-se que, no caso das redes de colaboração estabelecidas por docentes vinculados aos programas de Pós-Graduação em Contabilidade, quanto maior a pontuação 
obtida pelo docente no Qualis /CAPES (QUA) maior apresenta-se o círculo de interação (INT) em publicações científicas estabelecidas. A mesma tendência foi observada na relação entre a pontuação obtida pelo docente no Qualis /CAPES (QUA) com a quantidade de vínculos (VINC) de pesquisa.

Um aspecto que pode justificar a relação entre vínculos (VINC) e interações (INTE) é que em alguns casos ambas variáveis possuem o mesmo valor, ou seja, o docente estabelece uma única colaboração de pesquisa com cada um dos autores ou co-autores que compõe sua rede, explicando o elevado coeficiente obtido nessa relação, aproximando-se de uma correlação positiva perfeita nos três períodos.

As demais variáveis de pesquisa (quantidade de atores das redes e grau de centralização) são obtidas apenas por programa, por isso não foram objeto da análise de correlação.

Na Tabela 2, apresenta-se a quantidade de atores por programa. Observa-se que a maioria dos programas apresentou uma tendência crescente na quantidade de atores que compõem suas redes ao longo dos períodos de análise (FUCAPE, FURB, PUC SP, UERJ, UFBA, UFRJ, UFSC e UNISINOS), demonstrando que para esses programas ampliar a quantidade de colabores nas publicações é uma tática recorrente na produção científica docente. Dois programas apresentaram redução na quantidade de atores em suas redes ao longo do triênio (UNB/ UFPB/ UFRN e UniFECAP). No caso da primeira instituição, o ocorrido pode ter sido resultado da reestruturação do programa, que, em 2007, contava com docentes da UFPE. Já no caso da segunda, pode ser reflexo de uma constante reformulação no quadro de docentes ao longo do período, dificultando o estabelecimento de parcerias duradoras.

A UFC, por ser um programa recente, apresenta dados somente para o ano de 2009, não possibilitando um acompanhamento histórico das variáveis de pesquisa. Os demais programas apresentaram oscilações (aumento e diminuição) no número de atores ao longo do triênio (UFAM, UFMG, UFPE, UFPR, UPM, USP e USP RP). Curiosamente, nos sete programas com tendência oscilatória, o período em que as redes apresentaram maior número de atores foi 2008 , demonstrando que esse ano foi significativo para a colaboração científica de parte dos programas observados. Tal achado corrobora com a afirmação de Cruz et al. (2010) que menciona a formação de parcerias como uma prática frequente no meio científico.

Tabela 2: Análise da quantidade de atores das redes de colaboração

\begin{tabular}{l|c|c|c|c|l|c|c|c|c}
\hline \multicolumn{8}{c}{ Quantidade de atores das redes de colaboração } \\
\hline & $\mathbf{2 0 0 7}$ & $\mathbf{2 0 0 8}$ & $\mathbf{2 0 0 9}$ & Média & & $\mathbf{2 0 0 7}$ & $\mathbf{2 0 0 8}$ & $\mathbf{2 0 0 9}$ & Média \\
\hline FUCAPE & 30 & 43 & 45 & 39,33 & UFPR & 23 & 42 & 35 & 33,33 \\
\hline FURB & 52 & 80 & 112 & 81,33 & UFRJ & 34 & 50 & 58 & 47,33 \\
\hline PUC SP & 25 & 31 & 49 & 35,00 & UFSC & 50 & 52 & 79 & 60,33 \\
\hline UERJ & 19 & 23 & 34 & 25,33 & UNB/UFPB/UFRN & 69 & 51 & 50 & 56,67 \\
\hline UFAM & 33 & 60 & 59 & 50,67 & UniFECAP & 49 & 37 & 32 & 39,33 \\
\hline UFBA & 33 & 34 & 46 & 37,67 & UNISINOS & 36 & 42 & 46 & 41,33 \\
\hline UFC & - & - & 52 & 52,00 & UPM & - & 67 & 43 & 55,00 \\
\hline UFMG & 41 & 71 & 65 & 59,00 & USP & 93 & 95 & 75 & 87,67 \\
\hline UFPE & 46 & 60 & 51 & 52,33 & USP-RP & 33 & 50 & 42 & 41,67 \\
\hline
\end{tabular}

Fonte: Dados da Pesquisa 
Os programas da USP e da FURB, respectivamente, destacaram-se no triênio com as melhores médias de quantidade de atores, assim como também foram as instituições que apresentaram a maior quantidade de atores por período analisado: a USP, em 2007, com 93 atores e, em 2008, com 95 atores; e a FURB, em 2009, com 112 atores.

A análise da pontuação obtida por programa ao longo do triênio (Tabela 2) demonstra que o número de atores das redes pode ser visto como uma variável importante, no entanto não é a única que influencia no desempenho dos programas. Isso ficará mais evidente nas análises de vínculos e interações. No entanto, cabe ressalvar que os programas que se destacaram em quantidades de atores em suas redes de colaboração científica também são os que apresentaram maior média de pontos no Qualis/ CAPES, sendo a USP, em 2007, com 165,29, e, em 2008, com 120 pontos; e a FURB com uma média de 113,75 pontos em 2009. Quando se observa a quantidade de docentes em cada programa e seus respectivos desvios padrões, fica evidente a ocorrência de uma concentração de publicação em um número reduzido de docentes, ou seja, poucos docentes em praticamente todos os programas respondem por parcelas representativas da pontuação obtida pelos programas.

Tabela 3: Análise da pontuação obtida no Qualis/CAPES

\begin{tabular}{|c|c|c|c|c|c|c|c|c|c|c|c|c|}
\hline \multicolumn{13}{|c|}{ Estatística Descritiva da Pontuação no Qualis/ CAPES } \\
\hline & \multicolumn{4}{|c|}{2007} & \multicolumn{4}{|c|}{2008} & \multicolumn{4}{|c|}{2009} \\
\hline & $\mathbf{N}$ & Soma & Média & $\begin{array}{l}\text { Desvio } \\
\text { Padrão }\end{array}$ & $\mathbf{N}$ & Soma & Média & $\begin{array}{l}\text { Desvio } \\
\text { Padrão }\end{array}$ & $\mathbf{N}$ & Soma & Média & $\begin{array}{l}\text { Desvio } \\
\text { Padrão }\end{array}$ \\
\hline FUCAPE & 14 & 870 & 62,14 & 87,72 & 13 & 1390 & 106,92 & 110,03 & 17 & 1250 & 73,53 & 54,31 \\
\hline FURB & 13 & 1300 & 100,00 & 119,23 & 15 & 1550 & 103,33 & 94,24 & 16 & 1820 & 113,75 & 143,24 \\
\hline PUC SP & 12 & 440 & 36,67 & 44,99 & 12 & 400 & 33,33 & 46,77 & 12 & 310 & 25,83 & 50,35 \\
\hline UERJ & 11 & 150 & 13,64 & 19,12 & 12 & 140 & 11,67 & 15,28 & 18 & 310 & 17,22 & 48,00 \\
\hline UFAM & 10 & 460 & 46,00 & 128,86 & 13 & 830 & 63,85 & 109,28 & 16 & 710 & 44,38 & 139,09 \\
\hline UFBA & 11 & 470 & 42,73 & 48,39 & 12 & 200 & 16,67 & 31,43 & 13 & 770 & 59,23 & 98,78 \\
\hline UFC & - & - & - & - & - & - & - & - & 24 & 1080 & 45,00 & 80,49 \\
\hline UFMG & 12 & 800 & 66,67 & 48,87 & 12 & 1220 & 101,67 & 96,84 & 13 & 870 & 66,92 & 53,29 \\
\hline UFPE & 10 & 570 & 57,00 & 52,72 & 10 & 1290 & 129,00 & 117,52 & 13 & 1010 & 77,69 & 77,91 \\
\hline UFPR & 9 & 140 & 15,56 & 29,63 & 11 & 190 & 17,27 & 31,97 & 11 & 410 & 37,27 & 50,81 \\
\hline UFRJ & 12 & 480 & 40,00 & 46,51 & 14 & 360 & 25,71 & 39,75 & 14 & 950 & 67,86 & 115,17 \\
\hline UFSC & 11 & 930 & 84,55 & 122,83 & 12 & 1090 & 90,83 & 125,95 & 13 & 790 & 60,77 & 63,57 \\
\hline UNB/ UFPB/ UFRN & 21 & 770 & 36,67 & 38,25 & 15 & 830 & 55,33 & 56,43 & 15 & 710 & 47,33 & 77,41 \\
\hline UniFECAP & 12 & 970 & 80,83 & 81,40 & 15 & 510 & 34,00 & 49,68 & 12 & 420 & 35,00 & 30,00 \\
\hline UNISINOS & 10 & 730 & 73,00 & 75,14 & 10 & 820 & 82,00 & 90,65 & 10 & 1050 & 105,00 & 80,03 \\
\hline UPM & - & - & - & - & 10 & 740 & 74,00 & 58,54 & 11 & 1170 & 106,36 & 79,91 \\
\hline USP & 17 & 2810 & 165,29 & 113,53 & 20 & 2400 & 120,00 & 100,11 & 21 & 1690 & 80,48 & 70,46 \\
\hline USP-RP & 12 & 490 & 40,83 & 70,77 & 14 & 1090 & 77,86 & 67,50 & 13 & 1350 & 103,85 & 93,77 \\
\hline
\end{tabular}

Fonte: Dados da Pesquisa

A concentração da publicação docente fica clara ao se observar (Tabela 3) que a maior 
parcela dos desvios padrões dos programas na variável pontuação Qualis/CAPES são superiores à média, ou quando inferiores apresentam-se muito próximos da média, sendo que não foi detectado nenhum programa que não apresente concentração de publicação. O que acabou variando de um programa para o outro é intensidade dessa concentração.

O indício dessa concentração de publicação em poucos docentes pode ser observado na análise da quantidade de vínculos estabelecidos, da mesma forma que observado na pontuação no Qualis, os desvios padrões da quantidade de vínculos apresentam-se superiores à média de vínculos na maior parcela dos programas. Portanto na maioria dos casos observados, os docentes com elevada pontuação também se destacam na quantidade de vínculos estabelecidos nas redes de colaboração científica de seu respectivo programa.

Tabela 4: Análise dos vínculos das redes de colaboração

\begin{tabular}{|c|c|c|c|c|c|c|c|c|c|c|c|c|}
\hline \multicolumn{13}{|c|}{ Estatística Descritiva da Pontuação número de vínculos estabelecidos } \\
\hline & \multicolumn{4}{|c|}{2007} & \multicolumn{4}{|c|}{2008} & \multicolumn{4}{|c|}{2009} \\
\hline & $\mathbf{N}$ & Soma & Média & $\begin{array}{l}\text { Desvio } \\
\text { Padrão }\end{array}$ & $\mathbf{N}$ & Soma & Média & $\begin{array}{l}\text { Desvio } \\
\text { Padrão }\end{array}$ & $\mathbf{N}$ & Soma & Média & $\begin{array}{l}\text { Desvio } \\
\text { Padrão }\end{array}$ \\
\hline FUCAPE & 14 & 17 & 1,21 & 1,63 & 13 & 46 & 3,54 & 3,07 & 17 & 50 & 2,94 & 2,28 \\
\hline FURB & 13 & 65 & 5,00 & 4,40 & 15 & 86 & 5,73 & 5,55 & 16 & 131 & 8,19 & 6,86 \\
\hline PUC SP & 12 & 16 & 1,33 & 1,44 & 12 & 19 & 1,58 & 2,71 & 12 & 37 & 3,08 & 3,53 \\
\hline UERJ & 11 & 11 & 1,00 & 1,10 & 12 & 22 & 1,83 & 1,85 & 18 & 22 & 1,22 & 1,99 \\
\hline UFAM & 10 & 23 & 2,30 & 5,62 & 13 & 47 & 3,62 & 5,53 & 16 & 43 & 2,69 & 6,91 \\
\hline UFBA & 11 & 23 & 2,09 & 2,66 & 12 & 34 & 2,83 & 2,66 & 13 & 42 & 3,23 & 4,44 \\
\hline UFC & & - & - & - & - & - & - & - & 24 & 45 & 1,88 & 2,80 \\
\hline UFMG & 12 & 37 & 3,08 & 2,39 & 12 & 78 & 6,50 & 4,58 & 13 & 63 & 4,85 & 2,15 \\
\hline UFPE & 10 & 50 & 5,00 & 4,74 & 10 & 86 & 8,60 & 6,57 & 13 & 87 & 6,69 & 6,02 \\
\hline UFPR & 9 & 14 & 1,56 & 1,94 & 11 & 43 & 3,91 & 3,14 & 11 & 40 & 3,64 & 5,43 \\
\hline UFRJ & 12 & 26 & 2,17 & 3,10 & 14 & 40 & 2,86 & 3,68 & 14 & 56 & 4,00 & 5,48 \\
\hline UFSC & 11 & 49 & 4,45 & 4,93 & 12 & 78 & 6,50 & 6,76 & 13 & 111 & 8,54 & 7,03 \\
\hline UNB/UFPB/UFRN & 21 & 62 & 2,95 & 3,69 & 15 & 36 & 2,40 & 1,99 & 15 & 40 & 2,67 & 3,33 \\
\hline UniFECAP & 12 & 68 & 5,67 & 5,97 & 15 & 43 & 2,87 & 3,76 & 12 & 40 & 3,33 & 3,39 \\
\hline UNISINOS & 10 & 35 & 3,50 & 3,87 & 10 & 41 & 4,10 & 3,70 & 10 & 45 & 4,50 & 4,40 \\
\hline UPM & - & - & - & - & 10 & 63 & 6,30 & 4,85 & 11 & 59 & 5,36 & 3,14 \\
\hline USP & 17 & 105 & 6,18 & 3,13 & 20 & 102 & 5,10 & 3,42 & 21 & 72 & 3,43 & 2,79 \\
\hline USP-RP & 12 & 21 & 1,75 & 1,71 & 14 & 49 & 3,50 & 2,28 & 13 & 39 & 3,00 & 1,78 \\
\hline
\end{tabular}

Fonte: Dados da Pesquisa

Já na observação da quantidade de vínculos pela perspectiva dos programas (Tabela 4), observa-se uma tendência evolutiva em oito programas (FUCAPE, FURB, PUC SP, UFBA, UFPE, UFRJ, UFSC e UNISINOS), sendo que sete desses programas também apresentaram uma tendência de crescimento no número de atores que integram suas respectivas redes de colaboração. Seis programas demonstraram oscilação entre a evolução, a queda e a permanência do nível de vínculos firmados em suas redes de colaboração (UERJ, UFAM, UFMG, UFPR, UNB/ UFPB/ UFRN e USP RP); três programas apresentaram uma redução no número de vínculos (UniFECAP, UPM e USP); e o programa da UFC apenas possui dados relativos a 2009. 
Apesar de apresentar uma redução ao longo dos períodos em sua quantidade de vínculos, a USP apresentou a melhor média em 2007 (6,18 vínculos por docente); em 2008, o destaque ficou com a UFMG, com uma média de 6,50 vínculos; e, por fim, em 2009, com a UFSC, com uma média de 8,54 vínculos por docente. Já em quantidade total, o destaque, em 2007, continua com a USP, com 105 vínculos, e ainda com a USP, em 2008, com 102 vínculos; e, por fim, com a FURB, com 131 vínculos em 2009.

A Tabela 5 apresenta as análises das interações estabelecidas pelos docentes dos programas. Como observado anteriormente na análise de correlação, a quantidade de vínculos apresenta uma forte correlação positiva com a quantidade de interações nas publicações científicas, com coeficientes superiores a 0,90, com significância bicaudal. Ao comparar a quantidade de vínculos com as interações de pesquisa, verifica-se que a UERJ, nos anos de 2007 e 2008, apresentam valores de vínculos iguais aos de interações, assim como a UFRJ em 2008. Esse resultado demonstra que os docentes desses programas realizaram apenas uma colaboração com cada ator com o qual se apresenta ligado na rede.

Tabela 5: Análise das interações das redes de colaboração

\begin{tabular}{|c|c|c|c|c|c|c|c|c|c|c|c|c|}
\hline \multicolumn{13}{|c|}{ Estatística Descritiva da Pontuação número de interações nas publicações } \\
\hline & \multicolumn{4}{|c|}{2007} & \multicolumn{4}{|c|}{2008} & \multicolumn{4}{|c|}{2009} \\
\hline & $\mathbf{N}$ & Soma & Média & $\begin{array}{l}\text { Desvio } \\
\text { Padrão }\end{array}$ & $\mathbf{N}$ & Soma & Média & $\begin{array}{l}\text { Desvio } \\
\text { Padrão }\end{array}$ & $\mathbf{N}$ & Soma & Média & $\begin{array}{l}\text { Desvio } \\
\text { Padrão }\end{array}$ \\
\hline FUCAPE & 14 & 25 & 1,79 & 2,58 & 13 & 55 & 4,23 & 3,63 & 17 & 62 & 3,65 & 3,33 \\
\hline FURB & 13 & 76 & 5,85 & 6,26 & 15 & 106 & 7,07 & 6,87 & 16 & 170 & 10,63 & 9,88 \\
\hline PUC SP & 12 & 23 & 1,92 & 2,87 & 12 & 25 & 2,08 & 3,45 & 12 & 43 & 3,58 & 4,23 \\
\hline UERJ & 11 & 11 & 1,00 & 1,10 & 12 & 22 & 1,83 & 1,85 & 18 & 27 & 1,50 & 2,55 \\
\hline UFAM & 10 & 30 & 3,00 & 7,80 & 13 & 57 & 4,38 & 7,15 & 16 & 54 & 3,38 & 9,61 \\
\hline UFBA & 11 & 33 & 3,00 & 5,48 & 12 & 23 & 1,92 & 2,71 & 13 & 68 & 5,23 & 8,24 \\
\hline UFC & - & - & - & - & - & - & - & - & 24 & 54 & 2,25 & 3,52 \\
\hline UFMG & 12 & 44 & 3,67 & 3,23 & 12 & 84 & 7,00 & 7,37 & 13 & 71 & 5,46 & 2,76 \\
\hline UFPE & 10 & 56 & 5,60 & 4,88 & 10 & 126 & 12,60 & 12,99 & 13 & 107 & 8,23 & 8,30 \\
\hline UFPR & 9 & 17 & 1,89 & 2,47 & 11 & 45 & 4,09 & 3,39 & 11 & 55 & 5,00 & 8,60 \\
\hline UFRJ & 12 & 29 & 2,42 & 3,32 & 14 & 40 & 2,86 & 3,68 & 14 & 67 & 4,79 & 6,93 \\
\hline UFSC & 11 & 62 & 5,64 & 6,58 & 12 & 123 & 10,25 & 13,20 & 13 & 156 & 12,00 & 9,78 \\
\hline UNB/UFPB/UFRN & 21 & 63 & 3,00 & 3,73 & 15 & 40 & 2,67 & 2,41 & 15 & 46 & 3,07 & 4,51 \\
\hline UniFECAP & 12 & 94 & 7,83 & 9,86 & 15 & 48 & 3,20 & 4,52 & 12 & 44 & 3,67 & 4,03 \\
\hline UNISINOS & 10 & 40 & 4,00 & 4,42 & 10 & 56 & 5,60 & 5,82 & 10 & 54 & 5,40 & 5,80 \\
\hline UPM & - & - & - & - & 10 & 84 & 8,40 & 5,91 & 11 & 84 & 7,64 & 5,32 \\
\hline USP & 17 & 127 & 7,47 & 3,81 & 20 & 125 & 6,25 & 4,45 & 21 & 84 & 4,00 & 3,52 \\
\hline USP-RP & 12 & 22 & 1,83 & 1,95 & 14 & 60 & 4,29 & 3,73 & 13 & 59 & 4,54 & 3,45 \\
\hline
\end{tabular}

Fonte: Dados da Pesquisa

Portanto quanto maior a quantidade de interações em comparação à quantidade de vínculos, maior a reincidência de colaboração científica entre os docentes e os demais atores das redes. Nesse critério, os programas que apresentaram a maior ascensão entre a quantidade de vínculos e interações em 2007 foram a FUCAPE com 1,471 e a PUC SP, com 1,438 interações para cada vínculo; em 2008, foi a UFSC, com 1,577, e a UFPE, com 1,465; por sua vez, em 
2009, os destaques ficaram com a UFBA, com 1,619, e a USP RP, com 1,513 interações para cada vínculo estabelecido na sua respectiva rede de colaboração.

O programa que apresentou a maior quantidade total de interações foi a FURB, em 2009, com 170; nesse mesmo período, o mesmo programa apresentou também a maior quantidade absoluta de vínculos, 131 no total (Figura 1).

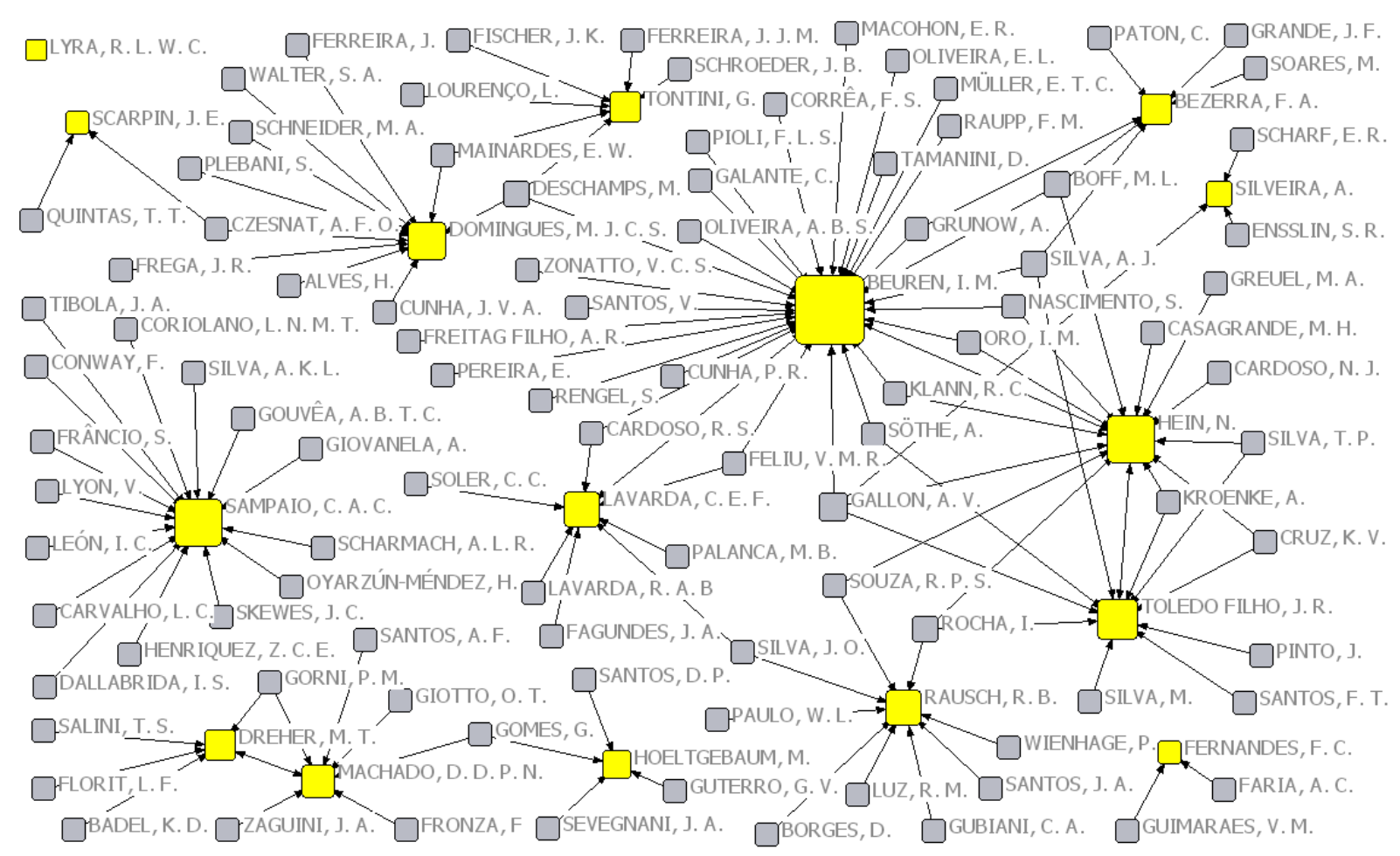

Figura 1: Rede de colaboração científica da FURB em 2009

No que diz respeito ao grau de centralização das redes de colaboração científica dos programas de Pós-Graduação em Contabilidade, podem-se observar os resultados na Tabela 6. Cabe ressaltar que o grau de centralização aqui demonstrado se refere ao grau de entrada, indicado pelo fluxo da rede e estabelecido apenas pelos docentes na presente pesquisa, tendo em vista que as redes foram identificadas em função destes.

Tabela 6: Grau de Centralização por programa

\begin{tabular}{l|c|c|c|c|l|c|c|c|c}
\hline & $\mathbf{2 0 0 7}$ & $\mathbf{2 0 0 8}$ & $\mathbf{2 0 0 9}$ & Média & & $\mathbf{2 0 0 7}$ & $\mathbf{2 0 0 8}$ & $\mathbf{2 0 0 9}$ & Média \\
\hline FUCAPE & $7,28 \%$ & $5,92 \%$ & $6,75 \%$ & $6,65 \%$ & UFPR & $12,50 \%$ & $9,91 \%$ & $12,83 \%$ & $11,75 \%$ \\
\hline FURB & $11,77 \%$ & $7,27 \%$ & $6,81 \%$ & $8,62 \%$ & UFRJ & $14,28 \%$ & $19,16 \%$ & $14,19 \%$ & $15,88 \%$ \\
\hline PUC SP & $7,88 \%$ & $11,70 \%$ & $6,98 \%$ & $8,85 \%$ & UFSC & $7,81 \%$ & $9,03 \%$ & $6,28 \%$ & $7,71 \%$ \\
\hline UERJ & $14,20 \%$ & $14,46 \%$ & $8,54 \%$ & $12,40 \%$ & UNB/UFPB/UFRN & $12,00 \%$ & $7,36 \%$ & $7,11 \%$ & $8,83 \%$ \\
\hline UFAM & $19,41 \%$ & $9,93 \%$ & $16,70 \%$ & $15,35 \%$ & UniFECAP & $9,95 \%$ & $18,13 \%$ & $19,36 \%$ & $15,81 \%$ \\
\hline UFBA & $19,34 \%$ & $11,43 \%$ & $6,95 \%$ & $12,57 \%$ & UNISINOS & $8,00 \%$ & $5,59 \%$ & $12,74 \%$ & $8,78 \%$ \\
\hline UFC & - & - & $7,31 \%$ & $7,31 \%$ & UPM & - & $6,06 \%$ & $5,71 \%$ & $5,88 \%$ \\
\hline UFMG & $5,72 \%$ & $6,61 \%$ & $5,24 \%$ & $5,86 \%$ & USP & $1,83 \%$ & $2,51 \%$ & $3,73 \%$ & $2,69 \%$ \\
\hline UFPE & $8,96 \%$ & $7,45 \%$ & $10,16 \%$ & $8,86 \%$ & USP-RP & $10,21 \%$ & $6,66 \%$ & $5,29 \%$ & $7,39 \%$ \\
\hline
\end{tabular}

Fonte: Dados da Pesquisa 
A interpretação do grau de centralização segue a seguinte lógica: quanto maior for o grau de centralização, corresponde à situação em que um determinado docente exerce um papel claramente central em relação aos demais atores, ou seja, que os fluxos de informações ou relações traçados na rede precisam passar pelo docente para que ocorram as ligações dentro da rede. Já o caso de um grau de centralização próximo de 0 implica a interpretação de que os atores estabelecem conexões diretas entre si, ou seja, não necessariamente precisam passar pelo mesmo docente para fechar os fluxos na rede.

Portanto as redes que possuem os graus de centralização mais elevados na análise sinalizam para uma concentração da maior parcela dos atores em uma quantidade reduzida de docentes. A menor média de centralização ocorreu na rede de colaboração dos docentes da USP (média de 2,69\%), e, em 2007, foi detectado o menor grau de centralização $(1,83 \%)$.

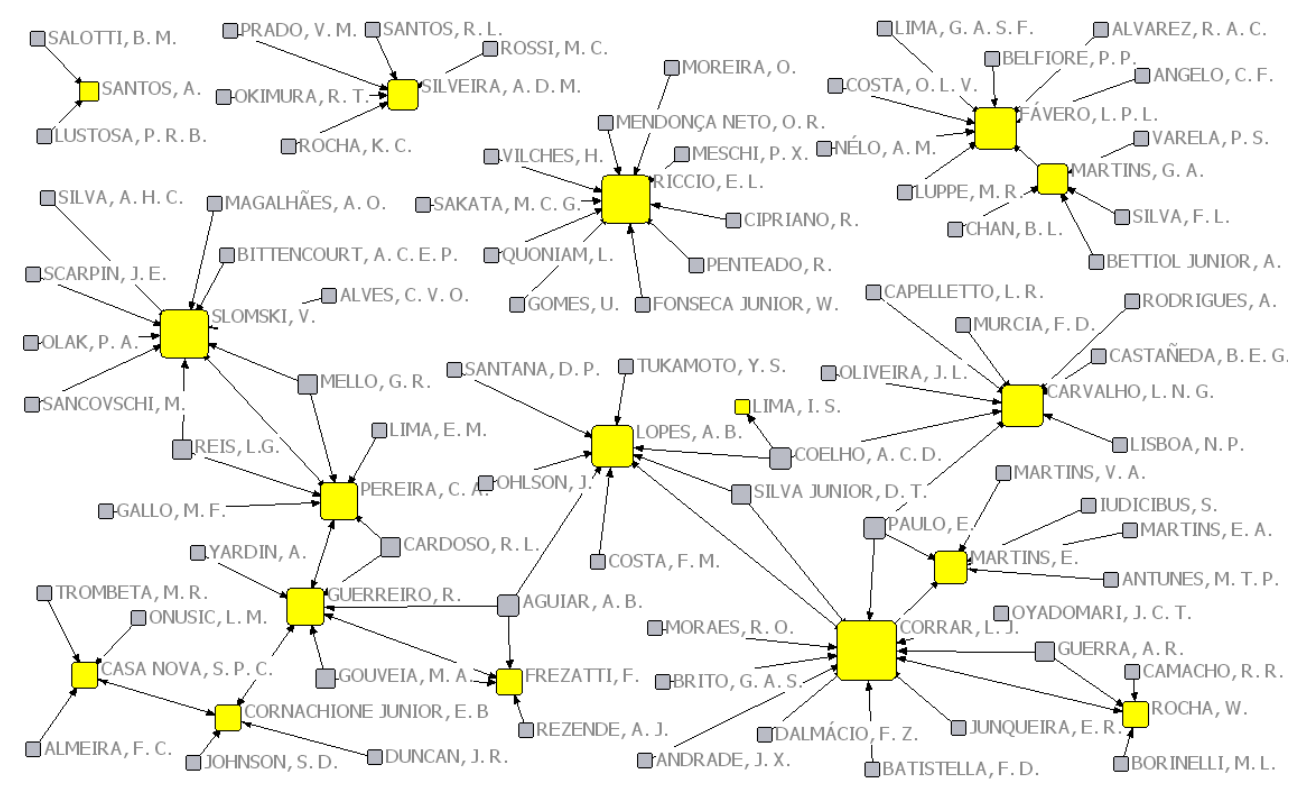

Figura 2: Rede de colaboração científica da USP em 2007

Ao observar a representação gráfica da rede de colaboração da USP referente a 2007, podemos verificar que não há existência de nós soltos, ou seja, todos os docentes estabeleceram vínculos no período. Podemos justificar o reduzido grau de centralização pela construção de relação quase que isolada pelos docentes do programa. Conforme observado na Tabela 6, o caso da USP é uma particularidade no presente estudo, tendo em vista que a média do grau de centralização dos programas apresenta-se superior aos 5\%.

Uma possível explicação para os elevados níveis de centralização detectados na presente pesquisa pode estar relacionada com uma tendência identificada na maior parcela dos programas: a concentração de vínculos de pesquisa em um número reduzido de docentes, ou mesmo a concentração de vínculos em apenas um docente. Essa concentração de vínculos pode indicar prestígio e desempenho diferenciado de alguns docentes diante de seus pares, porém demonstra também uma fragilidade na análise do programa, pois aponta para uma baixa diversificação de autores prolíficos em seu quadro.

A ocorrência de múltiplos casos de docente com a condição de nó solto na rede, ou seja, que não estabelece nenhum vínculo no período compreendido pela análise, pode ser outro fator que explica o nivelamento do grau de centralização para cima. 
Outro aspecto, observado e visto como preocupante pelos autores da presente pesquisa, ocorre quando os docentes de um determinado programa não estabelecem vínculos internos, ou seja, não apresentam parcerias em publicações de artigos em periódicos com os demais docentes do mesmo programa. Essa situação indica um isolamento da produção científica no programa, limitando a proliferação de redes sólidas de pesquisa no âmbito interno dos programas de PósGraduação em Contabilidade.

\section{CONCLUSÃO}

Esta pesquisa teve como objetivo mapear as redes de colaboração científica em periódicos dos docentes vinculados aos programas de Pós-Graduação em Contabilidade no triênio 20072009, identificando a quantidade de atores, vínculos, interações e grau de centralização das redes de colaboração dos programas. O mapeamento das redes de colaboração científica foi elaborado em função das seguintes variáveis: (i) quantidade de atores que compõem as redes; (ii) pontuação obtida no Qualis/CAPES; (iii) quantidade de vínculos traçados em cada programa; (iv) quantidade de interações de pesquisa estabelecidas nas redes; e, (v) grau de centralização das redes. Os autores da presente pesquisa justificam a relevância da identificação dos elementos das redes de colaboração por acreditarem que a interação entre os docentes (pesquisadores), proporcionada pela pesquisa gerada por esta parceria, é capaz de promover conhecimento e, consequentemente, evolução da área.

Com relação à quantidade de atores que compõem as redes de colaboração científica, observou-se uma evolução em todos os programas no triênio 2007-2009, evidenciando a preocupação dos programas de pós-graduação em melhorar seus resultados e o potencial de suas publicações científicas. Em relação à pontuação obtida no Qualis/CAPES, constatou-se que $66 \%$ dos programas apresentaram evolução na pontuação Qualis/CAPES no triênio, no entanto, convém destacar que os programas que apresentaram a maior média de pontuação por docente foram a USP, nos anos de 2007 e 2008, e a FURB, no ano de 2009.

Quanto à quantidade de vínculos por programa, 44\% dos programas apresentaram tendência evolutiva no triênio: $33 \%$ dos programas apresentaram oscilações entre evolução e queda e 16\% apresentaram redução. Apesar de apresentar uma queda na quantidade de vínculos, a USP se destaca no período de 2007 e de 2008, e a FURB no de 2009 por serem os programas que apresentaram a maior quantidade de vínculos no período. Com relação à quantidade de interações de pesquisa estabelecida pelas redes de colaboração, 39\% das instituições apresentaram evolução na quantidade de interações, com destaque para a FURB, no período de 2007 e 2009, e para a UFSC, no ano de 2008. No entanto, ao analisar de forma conjunta a quantidade de interações e de vínculos, os programas que se destacaram foram a FUCAPE, a UFSC e a UFBA. No que se refere ao grau de centralização das redes de colaboração científica, a instituição que possui a menor média de centralização é a USP, e a maior é a UFRJ.

Conclui-se que, de uma forma geral, os programas têm contribuído para a evolução das pesquisas e publicações científicas na área contábil. No entanto, essa contribuição poderia ser maior tendo em vista que se observa a concentração de publicações em um número reduzido de docentes. Mesmo sendo os programas de pós-graduação instrumentos formadores de pesquisadores, muitos docentes não têm se empenhado nessa função. Quanto à formação de redes de colaboração para o desenvolvimento de conhecimentos científicos, observa-se que a maioria dos programas tem-se utilizado de parcerias colaborativas no desenvolvimento de suas pesquisas. 
Recomenda-se para futuras pesquisas a replicação deste trabalho incluindo os anos de 2010 e 2011 e os artigos publicados nos eventos científicos da área.

\section{REFERÊNCIAS}

ALEJANDRO, V. A. O.; NORMAN, A. G. Manual introdutório à análise de Redes Sociais. UAEM - Universidad Autonoma Del Estado de México, 2005. 36 p.

BASTOS, A. V. B.; BORGES-ANDRADE, J. E. Nota Técnica. Cognição e ação: o ator ocupa a cena nos estudos organizacionais. In: CALDAS, Miguel; FACHIN, Roberto; FISCHER, Tânia (Organizadores da Edição Brasileira). Handbook de estudos organizacionais: ação e análise organizacionais. v. 3. São Paulo: Atlas, 2004, p. 69-76.

BEUREN, I. M. A. et al. Redes de pesquisa entre os egressos do Doutorado em Ciências Contábeis da FEA/USP. Revista Contabilidade, Gestão e Governança, Brasília, v. 12, n. 3, p. $72-86$ set./dez. 2009.

BEUREN, I. M.; SCHLINDWEIN, A. C.; PASQUAL, D. L. Abordagem da controladoria em trabalhos publicados no EnANPAD e no Congresso USP de Controladoria e Contabilidade de 2001 a 2006. Revista Contabilidade \& Finanças, São Paulo, v. 18, n. 45, p. 22-37, set./dez. 2007.

BORBA, J. A.; MURCIA, F. D. R. Oportunidade para pesquisa e publicação em Contabilidade: Um estudo preliminar sobre as revistas acadêmicas de Língua Inglesa do portal de periódicos da CAPES. Brazilian Business Review, Vitória, v. 3, n. 1, p. 88-103, jan./jun., 2006.

COORDENAÇÃO DE APERFEIÇOAMENTO DE PESSOAL DE NÍVEL SUPERIOR (CAPES). Documento de Área 2009. Disponível em: $<$ http://qualis.capes.gov.br/arquivos/ avaliacao/webqualis/criterios2007_2009/Criterios_Qualis_2008_27.pdf $>$ Acesso em: 2 fev. 2011.

COORDENAÇÃO DE APERFEIÇOAMENTO DE PESSOAL DE NÍVEL SUPERIOR (CAPES). Relação de cursos recomendados e reconhecidos. Disponível em: $<\mathrm{http}: / /$ conteudoweb.capes.gov.br/conteudoweb/ProjetoRelacaoCursosServlet?acao=pesquisarIes\&co digoArea $=60200006 \&$ descricaoArea $=$ CI $\%$ CANCIAS + SOCIAIS + APLICADAS $+\&$ descricao AreaConhecimento=ADMINISTRA $\% \mathrm{C} 7 \% \mathrm{C} 3 \mathrm{O} \&$ descricaoAreaAvaliacao $=$ ADMINISTRA $\%$ $\mathrm{C} 7 \% \mathrm{C} 3 \mathrm{O} \% 2 \mathrm{C}+\mathrm{CI} \% \mathrm{CANCIAS}+\mathrm{CONT} \% \mathrm{C} 1 \mathrm{BEIS}+\mathrm{E}+\mathrm{TURISMO}>$ Acesso em: 2 fev. $2011 \mathrm{a}$.

CRUZ,A.P.C. et al. Redes de cooperação entre pesquisadores do Congresso USP de Controladoria e Contabilidade: uma análise retrospectiva no período de 2001 a 2009. In: CONGRESSO USP DE CONTROLADORIA E CONTABILIDADE, 10., 2010, São Paulo. Anais... São Paulo: USP, 2010. CD-ROM.

ENSSLIN, S. R.; SILVA, B. M. S. Investigação do perfil dos artigos publicados nos Congressos de Contabilidade da USP e da USFC com ênfase na iniciação científica. Revista de Contabilidade e Organizações, São Paulo, v. 2, n. 3, p. 113-131, mai./ago. 2008.

ESPEJO, M. M. S. B. et al. Estado da Arte da pesquisa contábil: Um estudo bibliométrico de periódicos nacional e internacionalmente veiculados entre 2003 e 2007. Revista de Informação Contábil, Fortaleza, v. 3, n. 3, p. 94-116, jul./set. 2009.

FACHIN, O. Fundamentos de Metodologia. São Paulo: Saraiva, 2005. 210 p

GIL, A. C. Métodos e técnicas de pesquisa social. 5. ed. São Paulo: Atlas, 2010. 208 p. 
GUIMARÃES, T. A et al. A rede de programas de Pós-Graduação em Administração no Brasil: análise de relações acadêmicas e atributos de programas. Revista de Administração Contemporânea (RAC), Curitiba, v. 13, n. 4, p. 564-582, out./dez.2009.

KUHN, T. The Structure of Scientific Revolutions. Chicago: University of Chicago Press, 1970. $210 \mathrm{p}$.

LEITE FILHO, G. A. Padrões de produtividade de autores em periódicos e congressos na área de Contabilidade no Brasil: um estudo bibliométrico. In: CONGRESSO USP DE CONTROLADORIA E CONTABILIDADE, 6., 2006, São Paulo. Anais... São Paulo: USP, 2006. CD-ROM.

LYRIO, M. V.; BORBA, J. A.; COSTA, J. M. Controle gerencial: delineamento do perfil metodológico de uma amostragem de publicações acadêmicas nas áreas de Administração e Contabilidade de 2000 a 2004. Revista de Administração e Contabilidade da Unisinos (BASE), Rio Grande do Sul, v. 4, n. 2, p. 126-136, mai./ago. 2007.

MANAINARDES, E. et al. Produção científica brasileira em marketing de serviços: análise dos eventos EnANPAD e EMA, 2003-2006. Revista de Administração e Contabilidade da Unisinos (BASE), Rio Grande do Sul, v. 5, n. 2, p. 142-153, mai./ago. 2008.

MARION, J. C.; MARION, M. M. C. A importância da pesquisa no ensino da Contabilidade. Revista de Contabilidade do CRC/SP, n. 7; p. 05-20, mar./1998.

NASCIMENTO, S.; BEUREN, I. M. Redes Sociais na Produção Científica dos Programas de Pós-Graduação de Ciências Contábeis do Brasil. Revista de Administração Contemporânea (RAC), Curitiba, v. 15, n. 1, p. 4-66, jan./fev. 2011.

RICHARDSON, R. J. Pesquisa social: métodos e técnicas. São Paulo: Atlas, 1999. 334 p.

SILVA, A. C. B.; OLIVEIRA, E. C.; RIBEIRO FILHO, J. F. Revista contabilidade \& finanças - USP: uma comparação entre os períodos 1989/2001 e 2001/2004. Revista Contabilidade \& Finanças, São Paulo, n. 39, p. 20-32, set./dez. 2005.

SOARES, S. V.; EBSEN, K. S.; PFITSCHER, E. D. Formação acadêmica dos pesquisadores em Ciências Contábeis no Brasil: uma análise descritiva em níveis de doutorado, pós-doutorado e livre-docência. In: CONGRESSO USP DE INICIAÇÃO CIENTÍFICA EM CONTABILIDADE, 7., 2010, São Paulo. Anais... São Paulo: USP, 2010. CD-ROM.

SOUZA, F. C. et al. Análise das IES da área de Ciências Contábeis e de seus pesquisadores por meio de sua produção científica. Revista Vista e Revista, Belo Horizonte, v. 19, n. 3, p. 15-38, jul./set. 2008.

TEIXEIRA, E. B.; VITCEL, M. S.; LAMPERT, A. L. Iniciação científica: desenvolvendo competências e habilidades na formação do administrador. In: EnANPAD, 21.; 2007, Rio de Janeiro. Anais... Rio de Janeiro: EnANPAD, 2007. CD-ROM.

THEÓPHILO, C. R.; IUDÍCIBUS, S. de. Uma análise crítico-epistemológica da produção científica em Contabilidade no Brasil. UnB Contábil, Brasília, v. 8, n. 2, p. 15-38, jul./set. 2005.

TRIPODI, T.; FELLIN, P.; MEYER, H. J. Análise da pesquisa social: diretrizes para o uso de pesquisa em Serviço Social e Ciências Sociais. 2. ed. Rio de Janeiro: Francisco Alves, 1981. $338 \mathrm{p}$.

WALTER, S. A. et al. Uma análise da evolução do campo de ensino e pesquisa em Contabilidade sob a perspectiva de redes. Revista Universo Contábil, Blumenau, v. 5, n. 4, p. 76-93, out./ dez., 2009. 


\section{ENDEREÇO DOS AUTORES}

\section{Harley Almeida Soares da Silva}

Rua Paulino Júlio de Souza, 1663, Ipiranga

88.111-590 - São José - SC - Brasil

\section{Diane Rossi Maximiano Reina}

Rua: Julio D'acia Barreto, 500, Carvoeira

88.040-520 - Florianópolis - SC - Brasil

\section{Sandra Rolim Ensslin}

Rua Itararé, 41, Parque São Jorge

88.034-470 - Florianópolis - SC Brasil

\section{Donizete Reina}

Rua Julio D'acia Barreto, 500, Carvoeira

88.040-520 - Florianópolis - SC - Brasil 